\title{
Micromechanical Description of Curved Interfaces, Thin Films, and Membranes
}

\author{
I. Quasistatics \\ PETER A. KRALCHEVSKY \\ Laboratory of Thermodynamics and Physico-Chemical Hydrodynamics, \\ University of Sofia, Faculty of Chemistry, Sofia 1126, Bulgaria
}

Received November 17, 1987; accepted May 24, 1989

\begin{abstract}
Slow quasistatic deformations of a fluid interface or membrane are investigated theoretically from a micromechanical viewpoint. The surface rate-of-strain tensor is characterized by its invariants, $\alpha$ and $\beta$, which account for the interfacial dilation and shear, respectively. The macroscopic interfacial stretching and shearing tensions and the bending and torsion moments are expressed through integrals over the components of the pressure tensor. The results are applied to derive the Gibbsian fundamental thermodynamic equations of an arbitrarily curved interface from the fundamental equations of the adjacent bulk phases. Then, by means of a variational principle, the conditions for interfacial mechanical equilibrium are derived. 1990 Academic Press, Inc.
\end{abstract}

\section{INTRODUCTION}

According to Gibbs (1) the mechanical work for deformation of a fluid interface can be written in the form

$$
\delta w=\gamma \delta \alpha+B \delta H+\Theta \delta D .
$$

Here $\delta w$ is the elementary work of deformation per unit area, $\delta \alpha=[\delta(\Delta A)] / \Delta A$ is the relative increase in the area $\Delta A$ of the dividing surface, and

$$
H=\frac{1}{2}\left(c_{1}+c_{2}\right), \quad D=\frac{1}{2}\left(c_{1}-c_{2}\right),
$$

where $c_{1}$ and $c_{2}$ are the two principal curvatures of this surface; $H$ and $D$ are the mean and the deviatoric curvatures (the latter being a measure for the local deviation of the surface from the spherical shape). Equation [1.1] contains only intensive parameters and therefore it holds at each point of the surface. One sees from Eq. [1.1] that $\gamma \delta \alpha$ is the work for pure dilation $\left(\delta c_{1}=\delta c_{2}=0\right)$ of the interface, $B \delta H$ is the work for pure bending of the interface $\left(\delta c_{1}=\delta c_{2}, \delta \alpha=0\right)$, and $\Theta \delta D$ is the work for pure torsion of the interface $\left(\delta c_{1}\right.$ $=-\delta c_{2}, \delta \alpha=0$; see Fig. 1). That is why the quantities $B$ and $\Theta$ are called bending and torsion moments, respectively (2). $\gamma$ is the interfacial tension.

For a spherical interface $H=-1 / r_{0}, D=0$, and Eq. [1.1] reduces to the known expression (3-5)

$$
\delta w=\gamma \delta \alpha+\frac{B}{r_{0}^{2}} \delta r_{0},
$$

where $r_{0}$ is the radius of the dividing surface. From Eq. [1.3] Kondo (3) has derived in a thermodynamic way the generalized Laplace equation for a spherical interface,

$$
\frac{2 \gamma}{r_{0}}+\frac{B}{r_{0}^{2}}=P^{\mathrm{I}}-P^{\mathrm{II}}
$$

here $P^{\mathrm{I}}$ and $P^{\mathrm{II}}$ are the pressures in the two adjacent phases and

$$
B=r_{0}^{2}\left[\frac{\partial \gamma}{\partial r_{0}}\right],
$$

where $\left[\partial \gamma / \partial r_{0}\right]$ is a formal derivative, corresponding to changes in the radius of the di- 


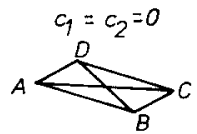

REFERENCE ST ATE

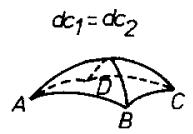

SMPLE BENDNG

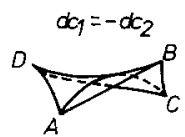

SIMPLE TORSION
FIG. 1. Bending and torsion modes of deformation of a surface element.

viding surface, $r_{0}$, at fixed physical state [ for details see Refs. (4-6)]. Obviously the bending moment, $B$, is zero at the Gibbsian surface of tension, where $\gamma$ has minimum value and the formal derivative in Eq. [1.5] is zero. When the interface is a thin liquid film intervening between two bulk phases, $\gamma$ is the film tension characterizing the film as a membrane of zero thickness $(5,6)$.

Tolman (7) and Buff (8) derived the following expressions for the interfacial tension $\gamma$ and bending moment $B$ of a spherical interface:

$$
\begin{array}{r}
\gamma=\int_{r_{\mathrm{I}}}^{r_{\mathrm{II}}}\left[\bar{P}-P_{\mathrm{T}}(r)\right] \frac{r^{2}}{r_{0}^{2}} d r, \\
B=\frac{2}{r_{0}} \int_{r_{\mathrm{I}}}^{r_{\mathrm{II}}}\left[\bar{P}-P_{\mathrm{T}}(r)\right]\left(r_{0}-r\right) r d r .
\end{array}
$$

Here $P_{\mathrm{T}}(r)$ is the tangential (with respect to the spherical dividing surface) component of the real nonisotropic pressure tensor,

$$
\underline{\mathbf{P}}=P_{\mathrm{N}} \mathbf{e}_{\mathrm{r}} \mathbf{e}_{\mathrm{r}}+P_{\mathrm{T}}\left(\mathbf{e}_{\theta} \mathbf{e}_{\theta}+\mathbf{e}_{\varphi} \mathbf{e}_{\varphi}\right)
$$

with $\mathbf{e}_{r}, \mathbf{e}_{\theta}$, and $\mathbf{e}_{\varphi}$ being the unit vectors of the local basis in spherical coordinates $r, \theta, \varphi ; \bar{P}$ is the isotropic pressure in the two adjacent phases:

$$
\begin{gathered}
\bar{P}=P^{\mathrm{I}} \theta\left(r_{0}-r\right)+P^{\mathrm{II}} \theta\left(r-r_{0}\right), \\
\theta(x)=1 \text { for } x>0 ; \\
=0 \text { for } x<0 .
\end{gathered}
$$

Eqs. [1.6] and [1.7] express the interfacial tension, $\gamma$, and the bending moment, $B$, as surface excess quantities. Indeed, $P_{\mathrm{T}}(r)$ is the tangential component of the real pressure tensor $\underline{\mathbf{P}}$, whereas $\bar{P}$ is the isotropic pressure in the idealized system, which is composed of two bulk phases and a mathematical dividing surface between them. The condition $B=0$ determines the so-called "surface of tension" (4).

Buff $(9,10)$ derived expressions of the type of Eqs. [1.6] and [1.7] for $\gamma, B$, and $\Theta$ in the general case of an arbitrarily curved interface. However, he used some approximations. Some years later Murphy (2) derived rigorous expressions for $\gamma, B$, and $\Theta$, but for the special case, when the pressure tensor $\underline{P}$ is transversely isotropic. (The definitions of the bending and torsion moments in the work of Murphy (2) are somewhat different from the definitions of Gibbs (1), which are used here.)

As pointed out by Evans and Skalak (11), the mechanical and thermodynamical properties of the biomembranes can be successfully described by means of a two-dimensional continuum, analogous to a Gibbs dividing surface. As discussed in Ref. (6) a thin liquid film can be described macroscopically as a membrane of zero thickness (the so-called "membrane approach"). Then it is possible to describe the mechanical properties of fluidliquid interfaces, thin liquid films, and biomembranes using the same formalism. The equations below in this paper are applicable to interfaces as well as to membranes. The system can be specified by substitution of an appropriate model expression for $\underline{\mathbf{P}}$ in the general equations.

Our purposes in this paper are:

(i) to continue the investigations of the previous authors $(2,9,10)$ and to derive expressions for $\gamma, B$, and $\Theta$ of more general validity;

(ii) to derive analogous expression for the shearing tension, $\zeta$, which can be important for subjects of the type of the biomembranes (11);

(iii) to derive the local fundamental equations of the interfacial thermodynamics from the respective equations in the bulk phases, paying special attention to the role of $\gamma, \zeta, B$, and $\theta$;

(iv) to derive the condition for interfacial mechanical equilibrium, generalizing Eq. 
[1.4], by means of a thermodynamic variational principle.

It is known that one must impose some physical conditions to define the position of the dividing surface [see, e.g., Refs. (4-6, 1214)]. Here and hereafter in this paper the dividing surface supposedly has been chosen in a certain way, irrespective of its concrete definition. The results thus obtained are valid for any choice of the dividing surface. The analysis of the dependence of $\gamma, \zeta, B$, and $\theta$ on the choice of the (arbitrarily curved) dividing surface is postponed to further investigations.

Despite the fact that we will finally arrive at some quasistatic relationships, it is convenient to work in the beginning with the rates of change of some quantities with time. Then it is easy to obtain the respective infinitesimal increments of these quantities during an elementary quasistatic process. For example, if $\delta t$ is a time increment, if $\underline{\Phi}$ is the space rateof-strain tensor, and if $\dot{H}$ is the time derivative of the mean curvature, $H$, then $\delta \underline{\Phi}=\underline{\Phi} \delta t$ is the space strain tensor and $\delta H=\dot{H} \delta t$ is the differential of $H$, which takes part in Eq. [1.1].

\section{SURFACE RATE-OF-STRAIN TENSOR}

Before studying the tensions in a membrane we will discuss briefly its deformations. The mathematical description of surface deformations has been worked out by Scriven (15), Aris (16), Eliassen (17), and Evans and Skalak (11). Some of their results are adapted and extended below according to our needs. The mathematical apparatus of the differential geometry is described in detail in Refs. (17-19).

\section{(a) Stretching and Shearing Parameters}

Let $\left(u^{1}, u^{2}\right)$ be curvilinear coordinates on the surface, and let $\mathbf{R}\left(u^{1}, u^{2}, t\right)$ be the running position vector of the dividing surface. Then

$$
\mathbf{a}_{\mu}=\frac{\partial \mathbf{R}}{\partial u^{\mu}}
$$

and

$$
\nabla_{\mathrm{II}}=\mathbf{a}^{\mu} \frac{\partial}{\partial u^{\mu}}
$$

are the vectors of the surface basis and the surface gradient operator. Here and hereafter the Greek indices take values 1 and 2, and summation over the repeating indices is carried out. It is known that the curvature tensor

$$
\underline{\mathbf{b}}=-\nabla_{\mathbf{I I}} \mathbf{n}
$$

( $\mathbf{n}$ is the running unit normal to the surface) is a symmetrical surface tensor, whose eigenvalues are the two principal curvatures, $c_{1}$ and $c_{2}$. Then it is clear that the trace of $\underline{\mathbf{b}}$ is equal to $2 H$ (cf. Eq. [1.2]) and the determinant of $\underline{\mathbf{b}}$ is the Gaussian curvature $K=c_{1} c_{2}$.

$$
\underline{\mathbf{U}}_{\mathrm{II}}=\mathbf{a}^{\mu} \mathbf{a}_{\mu} \quad \text { and } \quad \underline{\mathbf{q}}=\frac{1}{D}\left(\underline{\mathbf{b}}-H \underline{\mathrm{U}}_{\mathrm{II}}\right)
$$

are the surface idemfactor and curvature deviatoric tensor. The eigenvalues of $q$ are 1 and -1 , whereas both eigenvalues of $\underline{\mathbf{U}}_{\mathrm{II}}$ are equal to 1 . Therefore $\underline{\mathbf{U}}_{\mathrm{II}}$ and $\mathbf{q}$ are constant surface tensors; i.e., the covariant derivatives of their components are zero:

$$
a_{\lambda \mu, \nu}=q_{\lambda \mu, \nu}=0 \text {. }
$$

In particular, the components of $\underline{U}_{I I}$

$$
a_{\mu \nu}=\mathbf{a}_{\mu} \cdot \mathbf{a}_{\nu}
$$

are in fact the components of the surface metric tensor. In view of Eq. [2.4] the curvature tensor can be expressed as a sum of isotropic and deviatoric parts:

$$
b_{\lambda \mu}=H a_{\lambda \mu}+D q_{\lambda \mu} .
$$

Being a constant tensor in the regular points, $q$ is not defined in the ombilic points (where $\bar{D}=0$ ). However, in all equations of geometrical or physical importance $\mathbf{q}$ is multiplied by a factor, which tends to zero when approaching an ombilic point (cf. Eq. [2.7]) and the contribution of $\mathbf{q}$ is zero in these special points.

Let us suppose in addition that $\left(u^{1}, u^{2}\right)$ are the material surface coordinates [see Ref. (17) for details ]. Then

$$
\mathbf{v}=\left(\frac{\partial \mathbf{R}}{\partial t}\right)_{u^{1}, u^{2}}
$$

is the velocity of the surface material points. According to Eliassen (17) the surface rate-of 
(stretching)-strain tensor, $d_{\mu \nu}$, is defined by the expression

$$
\begin{aligned}
d_{\mu \nu} & =\frac{1}{2} \frac{d a_{\mu \nu}}{d t} \\
& =\frac{1}{2}\left(v_{\mu, \nu}+v_{\nu, \mu}-2 b_{\mu \nu} v^{[n]}\right),
\end{aligned}
$$

where

$$
v_{\mu}=\mathbf{a}_{\mu} \cdot \mathbf{v} \quad \text { and } \quad v^{[n]}=\mathbf{n} \cdot \mathbf{v}
$$

Let us consider the scalar quantities

$$
\begin{aligned}
\dot{\alpha} & =a^{\mu \nu} d_{\mu \nu}=\frac{1}{2} a^{\mu \nu} \frac{d a_{\mu \nu}}{d t} \\
& =a^{\mu \nu} v_{\mu, \nu}-2 H v^{[n]} \\
\dot{\beta} & =q^{\mu \nu} d_{\mu \nu}=\frac{1}{2} q^{\mu \nu} \frac{d a_{\mu \nu}}{d t} \\
& =q^{\mu \nu} v_{\mu, \nu}-2 D v^{[n]}
\end{aligned}
$$

(cf. Eqs. [2.7] and [2.9]). To find the meaning of $\alpha$ let us recall the identity $(16,17,20)$

$$
\frac{1}{2} a^{\mu \nu} \frac{d a_{\mu \nu}}{d t}=(a)^{-1 / 2} \frac{d}{d t}(a)^{1 / 2}
$$

where $a$ is the determinant of the surface metric tensor $a_{\mu \nu}$. If

$$
A(t)=\iint(a)^{1 / 2} d u^{1} d u^{2}
$$

is the area of a material surface parcel, then from Eqs. [2.11], [2.13], and [2.14] it follows that

$$
\frac{d A}{d t}=\iint \dot{\alpha}(a)^{1 / 2} d u^{1} d u^{2}
$$

(Note that $(a)^{1 / 2}$ is in fact the Jacobian of the surface curvilinear coordinates.) Hence $\dot{\alpha}$ is the rate of dilation (stretching) per unit area of the surface. Similarly, $\dot{\beta}$ represents the rate of shear per unit area (see Fig. 2). An alternative expression for $\dot{\alpha}$, which follows directly from Eqs. [2.2], [2.10], and [2.11] is

$$
\dot{\alpha}=\nabla_{\text {II }} \cdot \mathbf{v} \text {. }
$$

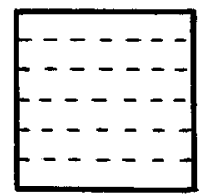

(a)

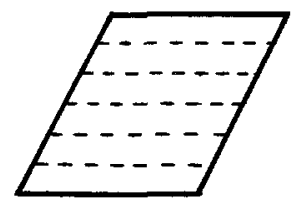

(b)
Fig. 2. A surface element before (a) and after (b) a deformation of shearing at constant area.

The total dilation and the total shear per unit area of the surface are (cf. Eqs. [2.11][2.13])

$$
\begin{aligned}
\alpha & =\int_{0}^{t} \dot{\alpha} d t=\frac{1}{2} \int_{0}^{t} a^{\mu \nu} \frac{d a_{\mu \nu}}{d t} d t \\
& =\frac{1}{2} \ln \left|\frac{a(t)}{a(0)}\right| \\
\beta & =\int_{0}^{t} \dot{\beta} d t=\frac{1}{2} \int_{0}^{t} q^{\mu \nu} \frac{d a_{\mu \nu}}{d t} d t,
\end{aligned}
$$

where $a(0)$ is the value of $a$ in the initial moment $t=0$. It is noteworthy that $\delta \alpha=\dot{\alpha} \delta t$ is the increment, which takes part in the righthand side of Eq. [1.1].

Equations [2.17] and [2.18] considerably simplify when the rate-of-strain tensor is diagonal in the basis of the principal curvatures all the time. (This is so, for example, in the familiar case of axisymmetric deformations of an axisymmetric membrane; see Refs. (11, 21).) Then the lines of curvature can serve as material coordinate lines on the surface. Due to the orthogonality of these coordinates one has

$$
\begin{gathered}
q^{11}=a^{11}=1 / a_{11}, \quad q^{22}=-a^{22}=-1 / a_{22} \\
a^{12}=a^{21}=q^{12}=q^{21}=0, \\
a_{12}=a_{21}=q_{12}=q_{21}=0, \quad[2.19]
\end{gathered}
$$

and one obtains from Eqs. [2.17] and [2.18]

$$
\begin{aligned}
& \alpha=\frac{1}{2} \ln \left|\frac{a_{11}(t) a_{22}(t)}{a_{11}(0) a_{22}(0)}\right|, \\
& \beta=\frac{1}{2} \ln \left|\frac{a_{11}(t) a_{22}(0)}{a_{1 \mathrm{I}}(0) a_{22}(t)}\right| .
\end{aligned}
$$


To elucidate the geometrical meaning of $\alpha$ and $\beta$ let us consider an elementary cell from the coordinate network, produced by the lines of curvature (see Fig. 3). Let $\delta s_{\mu}, \mu=1,2$, be the sides of this deformable surface element. It is known from the differential geometry (19), that

$$
\begin{aligned}
\delta s_{1}(t) & =\left(a_{11}\right)^{1 / 2} \delta u^{1}, \\
\delta s_{2} & =\left(a_{22}\right)^{1 / 2} \delta u^{2} .
\end{aligned}
$$

The circumferential extension ratios along the coordinate lines are

$$
\bar{\lambda}_{1}=\frac{\delta s_{1}(t)}{\delta s_{1}(0)}, \quad \bar{\lambda}_{2}=\frac{\delta s_{2}(t)}{\delta s_{2}(0)} .
$$

In view of Eqs. [2.21] and [2.22] one can transform Eqs. [2.20] into the following form:

$$
\alpha=\ln \left(\bar{\lambda}_{1} \bar{\lambda}_{2}\right), \quad \beta=\ln \left(\bar{\lambda}_{1} / \bar{\lambda}_{2}\right)
$$

One sees now that when the surface extension is isotropic $\left(\bar{\lambda}_{1}=\bar{\lambda}_{2}\right)$ the shearing parameter $\beta$ is zero, and vice versa, if an interfacial deformation at constant area $\left[\delta s_{1}(t) \delta s_{2}(t)\right.$ $=\delta s_{1}(0) \delta s_{2}(0)=$ const $]$ takes place, then $\bar{\lambda}_{1} \bar{\lambda}_{2}=1$ and $\alpha=0$.

Evans and Skalak (11) have proposed alternative parameters, $\bar{\alpha}$ and $\bar{\beta}$, characterizing the interfacial dilation and shear, which are connected with $\bar{\lambda}_{1}$ and $\bar{\lambda}_{2}$ as follows:

$$
\begin{aligned}
& \bar{\alpha}=\bar{\lambda}_{1} \bar{\lambda}_{2}-1, \\
& \bar{\beta}=\left(\bar{\lambda}_{1}^{2}+\bar{\lambda}_{2}^{2}\right) /\left(2 \bar{\lambda}_{1} \bar{\lambda}_{2}\right)-1 .
\end{aligned}
$$

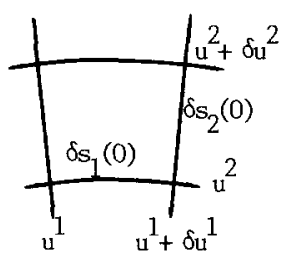

(a)

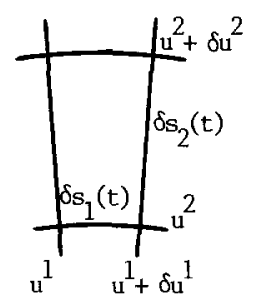

(b)
FIG. 3. Deformation of a surface element with sides of length $\delta s_{1}$ and $\delta s_{2}$ at the initial moment $t=0$ (a) and at a subsequent moment $t$ (b). The coordinate lines $u^{\alpha}$ $=$ const are lines of curvature.
From Eqs. [2.23] and [2.24] one obtains

$$
\begin{aligned}
& \bar{\alpha}=\exp (\alpha)-1, \\
& \bar{\beta}=\cosh (\beta)-1 .
\end{aligned}
$$

Hence $\bar{\alpha} \approx \alpha$ only for small deformations. In general, if $\delta \alpha$ in Eq. [1.1] was replaced by $\delta \bar{\alpha}$, the coefficient $\gamma$ in Eq. [1.1] would no longer have the meaning of interfacial tension. The problem is discussed in more detail in Ref. (21), where some applications of this formalism to deforming axisymmetric membranes are also given.

We will use below the stretching and shearing parameters, $\alpha$ and $\beta$, because of their simple relations with the rate-of-strain tensor (see Eqs. [2.11] and [2.12]).

For future reference we quote here also the equations

$$
\begin{aligned}
& \dot{H}=H_{, \mu} v^{\mu}+\left(H^{2}+D^{2}\right) v^{[n]} \\
&+\frac{1}{2} a^{\mu \nu} v_{, \mu \nu}^{[n]} \\
& \dot{D}=D_{, \mu} v^{\mu}+2 H D v^{[n]}+\frac{1}{2} q^{\mu \nu} v_{, \mu \nu}^{[n]}
\end{aligned}
$$

Eq. [2.26] was obtained by Eliassen (17), and Eq. [2.27] can easily be derived from Eliassen's expression for $\dot{K}\left(K=H^{2}-D^{2}\right.$ is the Gaussian curvature).

\section{(b) Examples}

We will consider now two simple examples which demonstrate the advantages of the formalism, illustrate the meaning, and give an idea for the values of some quantities, especially of the shearing parameter, $\beta$.

Example 1. Let

$$
z=z(x, t)
$$

be the equation which determines the shape of a sheet (say, of paper) at time, $t$ (see Fig. 4). The sheet is supposed to have translational symmetry about the axis $y$, which is orthogonal to the plane of the drawing. We suppose that at the initial moment, $t=0$, the sheet has been flat and lying in the plane $z=0$. Let the abscissa, $x$, and the ordinate, $y$, be the local surface coordinates. The material points on 


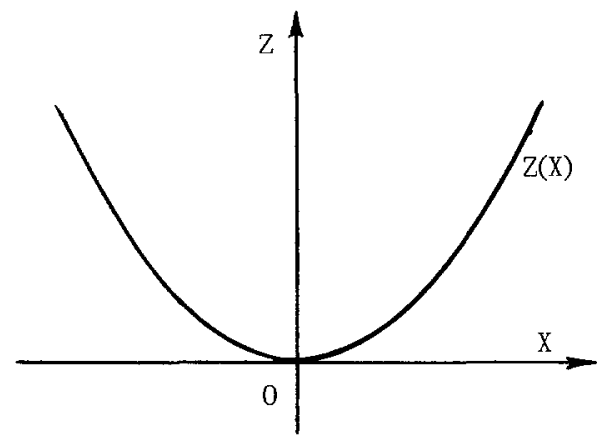

FIG. 4. Shape of a deforming sheet, which has translational symmetry along the $y$-axis.

the sheet are numerated by their coordinates at the initial moment $t=0: u^{1}=x_{0}, u^{2}=y_{0}$. During the deformation one has

$$
x=x\left(x_{0}, t\right), y=y_{0} .
$$

Let $\mathbf{i}, \mathbf{j}$, and $\mathbf{k}$ be the unit vectors along the axes $x, y$, and $z$. Then the position vector of a point of the sheet is

$$
\mathbf{R}=\mathbf{i} x+\mathbf{j} y+\mathbf{k} z(x, t) .
$$

Then from Eq. [2.1] one obtains

$$
\begin{aligned}
& \mathbf{a}_{1}=\frac{\partial \mathbf{R}}{\partial x_{0}}=\mathbf{i} \frac{\partial x}{\partial x_{0}}+\mathbf{k} z^{\prime} \frac{\partial x}{\partial x_{0}} \\
& \mathbf{a}_{2}=\frac{\partial \mathbf{R}}{\partial y_{0}}=\mathbf{j}, \quad z^{\prime}=\left(\frac{\partial z}{\partial x}\right)_{t} .
\end{aligned}
$$

Eqs. [2.6] and [2.31] lead to

$$
\begin{gathered}
a_{11}(t)=\left(1+z^{\prime 2}\right)\left(\frac{\partial x}{\partial x_{0}}\right)^{2}, \\
a_{22}(t)=1,
\end{gathered}
$$

and the mixed components of $a_{\mu \nu}$ are equal to zero. The values of the respective components at the initial moment are

$$
\begin{aligned}
& a_{11}(0)=a_{22}(0)=1, \\
& a_{12}(0)=a_{21}(0)=0 .
\end{aligned}
$$

The sheet is incompressible. Hence $\alpha=0$ and it follows from Eq. [2.20] that

$$
a_{11}(t) a_{22}(t)=a_{11}(0) a_{22}(0) \text {. }
$$

By substituting Eqs. [2.32] and [2.33] into [2.34] and subsequent integration one finds the explicit form of the function $x_{0}$ $=x_{0}(x, t)$ :

$$
x_{0}(x, t)=\int_{0}^{x}\left(1+z^{\prime 2}\right)^{1 / 2} d x .
$$

Moreover, it follows from Eqs. [2.20], [2.32], [2.33], and [2.34] that not only $\alpha$, but also $\beta$ is identically zero during the deformation. Although intuitively transparent this result is by no means trivial, because neither the curvatures $H$ and $D(H=D$ due to the translational symmetry) nor the surface velocity field

$$
v=i\left(\frac{\partial x}{\partial t}\right)_{x_{0}}+k\left(\frac{\partial z}{\partial t}\right)_{x_{0}}
$$

in the right-hand side of Eqs. [2.11] and [2.12] is equal to zero.

Example 2. Let us consider the deformation. of a sphere into a biconcave disk at constant area. [Similar deformations are observed in experiments with red blood cells (11).] It is convenient to use spherical coordinates $r, \theta$, $\varphi$. The equation of the surface is

$$
\begin{aligned}
\mathbf{R}= & \mathbf{i} r(\theta) \sin \theta \cos \varphi \\
& +\mathbf{j} r(\theta) \sin \theta \sin \varphi+\mathbf{k} r(\theta) \cos \theta
\end{aligned}
$$

with

$$
\begin{aligned}
& r^{2}(\theta)=b^{2} \cos ^{2} \theta+c^{2} \sin ^{2} \theta \\
& b<c, \quad 0<\theta<\pi
\end{aligned}
$$

(see Fig. 5). We choose $\theta$ and $\varphi$ as local surface coordinates. In the initial moment $t=0$ the surface was a sphere of radius $R(b=c=R)$. The surface material points are numerated by their coordinates $u^{1}=\theta_{0}$ and $u^{2}=\varphi_{0}$ at the initial moment. Similarly to [2.32] one derives

$$
\begin{aligned}
& a_{11}=\left(\frac{\partial \theta}{\partial \theta_{0}}\right)^{2}\left(r^{2}+r^{2}\right), \\
& a_{22}=r^{2} \sin ^{2} \theta, \quad r^{\prime}=\frac{d r}{d \theta}
\end{aligned}
$$




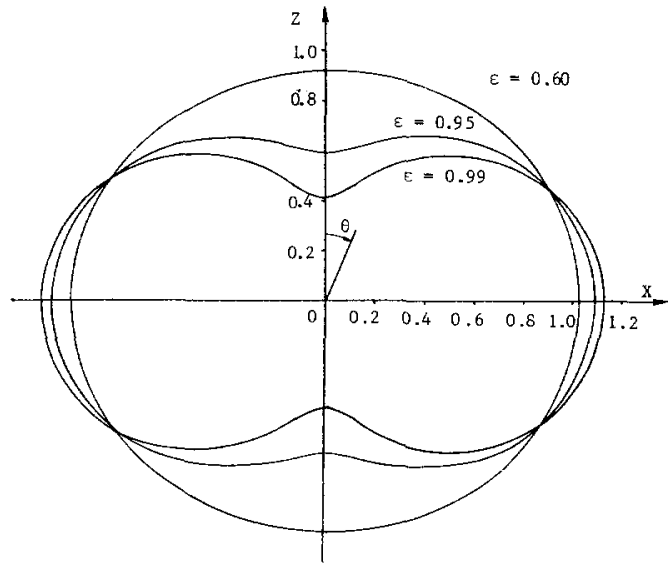

FIG. 5. Deformation of a sphere into a biconcave disk. $\theta$ is the polar angle and $\epsilon^{2}=1-b^{4} / c^{4}$ (cf. Eq. [2.37]).

the other components of the tensor $a_{\mu \nu}$ are zero. In the initial moment

$$
a_{11}(0)=R^{2}, \quad a_{22}(0)=R^{2} \sin ^{2} \theta_{0} .
$$

The instantaneous area of the surface is

$$
\begin{gathered}
A=2 \pi c^{2}\left[\left(1-\epsilon^{2}\right)^{1 / 2}+\frac{1}{\epsilon} \arcsin (\epsilon)\right], \\
\epsilon^{2}=1-b^{4} / c^{4}
\end{gathered}
$$

The incompressibility condition, Eq. [2.34], along with Eqs. [2.37], [2.38], and the boundary condition $\left.\theta_{0}\right|_{\theta=\pi / 2}=\pi / 2$, leads to

$$
\begin{array}{r}
\cos \theta_{0}=\frac{1}{2 p}\left[\left(1-\epsilon^{2} \cos ^{2} \theta\right)^{1 / 2} \cos \theta\right. \\
\left.+\frac{1}{\epsilon} \arcsin (\epsilon \cos \theta)\right],
\end{array}
$$

where

$p=\frac{R^{2}}{c^{2}}=\frac{1}{2}\left[\left(1-\epsilon^{2}\right)^{1 / 2}+\frac{1}{\epsilon} \arcsin (\epsilon)\right]$.

The latter equation follows from Eq. [2.40]. Finally, Eqs. [2.20] and [2.34] yield

$$
\beta=\ln \left[a_{22}(0) / a_{22}(t)\right],
$$

and then from Eqs. [2.37-2.40] and [2.42] one obtains

$$
\beta=\ln \frac{p \sin ^{2} \theta_{0}}{\left[\sin ^{2} \theta+\left(1-\epsilon^{2}\right)^{1 / 2} \cos ^{2} \theta\right] \sin ^{2} \theta} .
$$

Eqs. [2.41] and [2.43] determine $\beta$ as a function of $\theta$. At the initial moment $\epsilon=0, p=1$, $\theta=\theta_{0}$, and $\beta$ is identically zero. When $0<\epsilon$ $<\sqrt{3} / 2, \beta$ is a function of $\theta$, which takes negative values. If $\epsilon>\sqrt{3} / 2$ a dimple appears at the center of the disk and $\beta$ is positive in the proximity of the dimple (see Fig. 6).

\section{ELEMENTARY WORK OF INTERFACIAL DEFORMATION}

\section{(a) Micromechanical Derivation}

As mentioned earlier, the continuous and nonisotropic real pressure tensor $\underline{\mathbf{P}}$ is replaced in the idealized system by an isotropic tensor

$$
\underline{\overline{\mathbf{P}}}=\bar{P} \underline{\mathbf{U}}, \quad \bar{P}=P^{\mathrm{l}} \theta(-\lambda)+P^{\mathrm{II}} \theta(\lambda),
$$

which is discontinuous at the dividing surface. $P^{\mathrm{I}}$ and $P^{\mathrm{II}}$ are the values of the isotropic pressures in the two neighboring bulk phases of the idealized system and $\underline{\mathbf{U}}$ is the space idem-

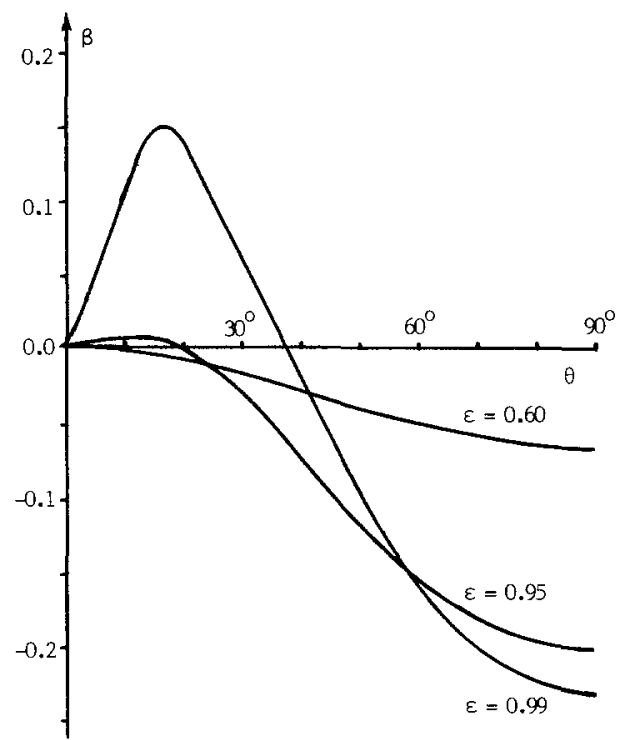

FIG. 6. Plots of the shearing parameter, $\beta$, vs polar angle, $\theta$, for the three axisymmetric surfaces shown in Fig. 5 . 
factor. The mechanical work, done for deformation of the interface during a small time interval $\delta t$, can be expressed as an excess quantity (5):

$$
\int_{A} \delta w d A=-\int_{V}(\underline{\mathbf{P}}-\underline{\overline{\mathbf{P}}}):(\underline{\Phi} \delta t) d V .
$$

Here $\delta w$ is the work of interfacial deformation per unit area of the dividing surface, $d A$ $=a^{1 / 2} d u^{1} d u^{2}$ is a surface element, ":" denotes double scalar product of two tensors, and $\underline{\Phi}$ is the space rate-of-strain tensor:

$$
\underline{\Phi}=\frac{1}{2}\left[\nabla \mathbf{w}+(\nabla \mathbf{w})^{+}\right] .
$$

Here $w$ is the velocity field in the bulk phases and "+" denotes conjugation. For slow quasistatic processes we will use Eliassen's (17) kinematical restriction

$$
\mathbf{w}=\mathbf{v}-\lambda\left(\nabla_{\mathrm{II}} \mathbf{v}\right) \cdot \mathbf{n},
$$

where $\lambda$ is the perpendicular distance from the point in the space to the dividing surface. The three coordinates $\left(u^{1}, u^{2}, \lambda\right)$ form a curvilinear coordinate network in space. This network moves together with the deforming dividing surface. Eq. [3.4] states that each material point is fixed at a point of the moving coordinate network $\left(u^{1}, u^{2}, \lambda\right)$. In particular, each layer of material points, corresponding to $\lambda$ $=$ const at a given time moment, remains parallel to the dividing surface during the further deformation. The integration in the left-hand side of Eq. [3.2] is carried out over an arbitrary parcel of the dividing surface and the integration in the right-hand side-over the respective volume shown in Fig. 7. The latteral surface of this volume is orthogonal to the dividing surface. The vectorial element $d s$ on the lateral surface can be expressed in the form (17)

$$
d \mathbf{s}=\mathbf{v} \cdot \underline{\mathbf{L}} d \lambda d l,
$$

where $d l$ is an arc element on the contour $C$ (see Fig. 7), $v$ is the outer running unit normal to $C$ in the dividing surface, and

$$
\underline{\mathbf{L}}=(1-2 \lambda H) \underline{\mathbf{U}}_{\mathrm{II}}+\lambda \underline{\mathbf{b}}
$$

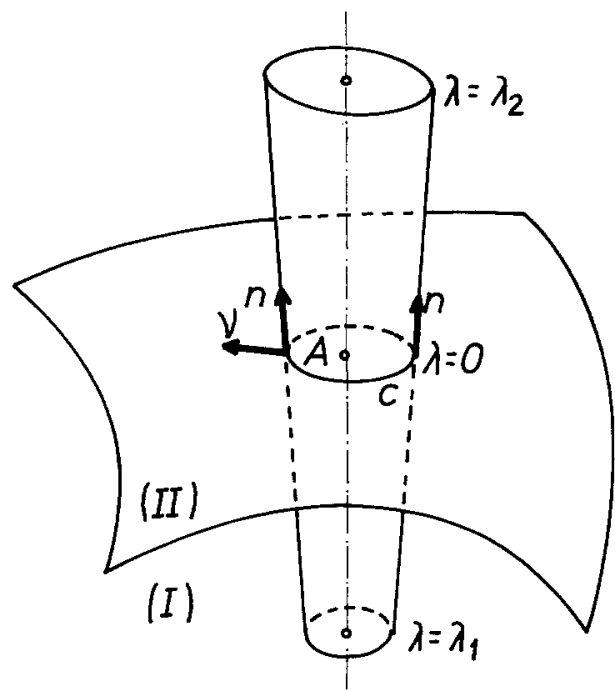

FIG. 7. An imaginary cylinder whose lateral surface is orthogonal to the dividing surface (between phases I and II) and whose bases are parallel to it. $\mathbf{n}$ and $\nu$ are running normals to the dividing surface and to the contour $C$, respectively.

is a surface tensor. Similarly to Eq. [3.5] one can express the volume element in Eq. [3.2] in the form $(17,22)$

$$
\begin{aligned}
d V & =\chi d A d \lambda, \\
\chi & =(1-\lambda H)^{2}-\lambda^{2} D^{2} .
\end{aligned}
$$

The substitution of Eq. [3.4] in Eq. [3.3] leads to

$$
\begin{aligned}
\underline{\Phi}= & \frac{1}{2 \chi}[(1-2 \lambda H) \\
& \times\left(w_{\mu, \nu}+w_{\nu, \mu}\right)+\lambda\left(w_{\mu, \sigma} b_{\nu}^{\sigma}+w_{\nu, \sigma} b_{\mu}^{\sigma}\right) \\
& \left.\quad-2\left(b_{\mu \nu}-\lambda K a_{\mu \nu}\right) v^{[n]}\right] \mathbf{a}^{\mu} \mathbf{a}^{\nu}
\end{aligned}
$$

The derivation of Eq. [3.8] is given in the Appendix. Introducing the tensor

$$
\underline{\mathbf{p}}^{x}=\underline{\mathbf{P}}-\underline{\overline{\mathbf{p}}},
$$

one obtains from Eqs. [3.2] and [3.7]

$$
\int_{A} \delta w d A=-\int_{V} \underline{\mathbf{P}}^{\chi}: \underline{\mathbf{\Phi}} \delta t \chi d \lambda d A .
$$

Then using the arbitrariness of the parcel $A$ one obtains from Eq. [3.10] the expression 


$$
\delta w=-\int_{\lambda_{1}}^{\lambda_{2}} \underline{\mathbf{P}}^{\chi}: \underline{\boldsymbol{\Phi}} \delta t \chi d \lambda .
$$

Further simplifications in the right-hand side of Eq. [3.11] are possible only if some information about the nature of the process is available. Let the surface tensor $\Phi$ be expressed in the form

$$
\underline{\mathbf{\Phi}}=\frac{1}{2}\left(\underline{\mathbf{U}}_{\mathrm{II}}: \underline{\mathbf{\Phi}}\right) \underline{\mathbf{U}}_{\mathrm{II}}+\frac{1}{2}(\underline{\mathbf{q}}: \underline{\mathbf{\Phi}}) \underline{\mathbf{q}} .
$$

Eq. [3.12] means that the tensor $\underline{\Phi}$ is diagonal in the basis of the principal curvatures. In other words, the curvature of the dividing surface is in correspondence with the deformations in the adjacent phases or vice versa.

From Eq. [3.12] it follows directly that

$$
\underline{\mathbf{P}}^{\chi}: \underline{\Phi}=\frac{1}{2} \underline{\mathbf{P}}^{\chi}:\left[\underline{\mathrm{U}}_{\mathrm{II}}\left(\underline{\mathrm{U}}_{\mathrm{II}}: \underline{\Phi}\right)+\underline{\mathbf{q}}(\underline{\mathbf{q}}: \underline{\Phi})\right] \text {. }
$$

The same equation [3.13] can be obtained under another assumption. If the real pressure tensor has the form

$$
\begin{aligned}
\underline{\mathbf{P}}=\frac{1}{2}\left(\underline{\mathbf{U}}_{\mathrm{II}}: \underline{\mathbf{P}}\right) \underline{\mathbf{U}}_{\mathrm{II}} \\
+\frac{1}{2}(\underline{\mathbf{q}}: \underline{\mathbf{P}}) \underline{\mathbf{q}}+P_{N} \mathbf{n n},
\end{aligned}
$$

then Eq. [3.13] holds without any limitations on the form of $\underline{\Phi}$. The representation [3.14], which was introduced in slightly different (but equivalent) form by $\operatorname{Buff}(9,10)$, means that at each point of the material continuum two of the eigenvectors of $\underline{P}$ are directed along the two lines of curvature of the surface $\lambda=$ const passing through the same point. The third eigenvector is always orthogonal to the dividing surface. These properties of $\mathbf{P}$ in the space can be thought of as being induced by the geometry of the interface.

Some computations, included in the Appendix, yield

$$
\begin{aligned}
\underline{\mathbf{U}}_{\mathrm{II}}\left(\underline{\mathbf{U}}_{\mathrm{II}}: \underline{\mathbf{\Phi}}\right) & +\underline{\mathbf{q}}(\underline{\mathbf{q}}: \underline{\mathbf{\Phi}})=\frac{1}{\chi}\left(\chi \underline{\mathbf{U}}_{\mathrm{II}} \dot{\alpha}+\chi \underline{\mathbf{q}} \dot{\beta}\right. \\
& -2 \lambda \underline{\mathbf{L}} \dot{H}-2 \lambda \underline{\mathbf{q}} \cdot \underline{\mathbf{L}} \dot{D}), \quad[3.15
\end{aligned}
$$

where, as usual, the dot symbolizes time derivative. The substitution from Eqs. [3.13] and [3.15] into Eq. [3.11] leads to

$$
\delta w=\gamma \delta \alpha+\zeta \delta \beta+B \delta H+\Theta \delta D
$$

with

$$
\begin{aligned}
\delta \alpha=\dot{\alpha} \delta t, & \delta \beta=\dot{\beta} \delta t, \\
\delta H=\dot{H} \delta t, & \delta D=\dot{D} \delta t
\end{aligned}
$$

and

$$
\begin{gathered}
\gamma=-\frac{1}{2} \int_{\lambda_{1}}^{\lambda_{2}} \underline{\mathbf{U}}_{\mathrm{II}}: \underline{\mathbf{P}}^{\chi} \chi d \lambda \\
\zeta=-\frac{1}{2} \int_{\lambda_{1}}^{\lambda_{2}} \underline{\mathbf{q}}: \underline{\mathbf{P}}^{\chi} \chi d \lambda, \\
B=\int_{\lambda_{1}}^{\lambda_{2}} \underline{\mathbf{L}}: \underline{\mathbf{P}}^{\chi} \lambda d \lambda \\
\Theta=\int_{\lambda_{1}}^{\lambda_{2}}(\underline{\mathbf{q}} \cdot \underline{\mathbf{L}}): \underline{\mathbf{P}}^{\chi} \lambda d \lambda .
\end{gathered}
$$

Eq. [3.16] gives the sought-for expression for the mechanical work of interfacial deformation. $\delta \alpha$ is the dilation of the dividing surface and $\gamma$ is the interfacial (stretching) tension; $\delta \beta$ measures the elementary shearing of the dividing surface and $\zeta$ is the interfacial shearing tension. $\delta H$ and $\delta D$ represent the elementary bending and torsion of the interface, and $B$ and $\theta$ are the corresponding interfacial bending and torsion moments.

\section{(b) Discussion}

Equation [3.16] expresses the interfacial deformation as a superposition of dilation, shear, bending, and torsion. A comparison of Eq. [3.16] with Gibbs' equation [1.1] shows that a new term, $\zeta \delta \beta$, appears. For a common liquid interface this term is usually neglected. However, as pointed out by Evans and Skalak (11), the shearing effect is very important for biological membranes, which often deform at constant area $(\delta \alpha=0)$. If the pressure tensor $\underline{P}$ is transversely isotropic, i.e., if

$$
\underline{\mathbf{P}}=P_{\mathrm{T}} \underline{\mathbf{U}}_{\mathrm{II}}+P_{N} \mathbf{n} \mathbf{n}
$$

it follows from Eq. [3.18] that $\zeta$ is identically zero. Hence Eq. [3.20] should not be applied when describing subjects like biomembranes. 
The stretching, bending, and torsion effects have been investigated by Murphy (2), who have used the condition for transversal isotropy [3.20]. By substitution of Eq. [3.20] in Eqs. [3.18] and [3.19] one can derive the results of Murphy as a special case. (Murphy has used $\delta H$ and $\delta K$ as independent variations. We preferred to use here the Gibbs variables $\delta H$ and $\delta D$ because of their geometrical transparency (see Fig. 1) and physical convenience: they lead to coefficients $B$ and $\theta$ having one and the same physical dimension.) Sometimes it is convenient to introduce the quantities

$$
\gamma_{1}=\gamma+\zeta \text { and } \gamma_{2}=\gamma-\zeta
$$

(It is shown in Ref. (6) that $\gamma_{1}$ and $\gamma_{2}$ are the eigenvalues of the hydrostatic surface stress tensor at the surface of tension.) Moreover, one can define

$$
\begin{gathered}
B_{1}=\frac{1}{2}(B+\theta), \quad B_{2}=\frac{1}{2}(B-\boldsymbol{\theta}), \\
P_{\mathrm{T} 1}=\frac{1}{2}\left(\underline{\mathbf{U}}_{\mathrm{II}}+\underline{\mathbf{q}}\right): \underline{\mathbf{P}}, \\
P_{\mathrm{T} 2}=\frac{1}{2}\left(\underline{\mathbf{U}}_{\mathrm{II}}-\underline{\mathbf{q}}\right): \underline{\mathbf{P}} .
\end{gathered}
$$

Then one easily derives from Eqs. [3.7] and [3.18] that

$$
\begin{array}{r}
\gamma_{i}=\int_{\lambda_{1}}^{\lambda_{2}}\left(\bar{P}-P_{\mathrm{T} 1}\right)\left(1-2 \lambda H+\lambda^{2} K\right) d \lambda, \\
i=1,2 .
\end{array}
$$

Similarly, it follows from Eqs. [2.7], [3.6], and [3.19] that

$$
\begin{aligned}
B_{i}= & \int_{\lambda_{1}}^{\lambda_{2}}\left(P_{\mathrm{T} 1}-\bar{P}\right)\left\{\lambda-\lambda^{2}\right. \\
& \left.\times\left[H+(-1)^{i} D\right]\right\} d \lambda, \quad i=1,2 .[3.25]
\end{aligned}
$$

If one can neglect the contribution of the longrange interactions in the integrals [3.24] and [3.25] (it is not always possible), one can drop out the terms with $\lambda^{2}$ and thus the results of Buff $(9,10)$ are obtained as approximate versions of Eqs. [3.24] and [3.25]. (Buff has denoted $B_{i}, i=1,2$, by $C_{i} / s$ and called them "the intensive curvature terms.")
For a spherical dividing surface with radius $r_{0}$ one has $H=-1 / r_{0}, D=0, \zeta=0, \Theta=0$, $P_{\mathrm{T} 1}=P_{\mathrm{T} 2}, \gamma_{1}=\gamma_{2}=\gamma, B_{1}=B_{2}=B / 2$ and it follows from Eqs. [3.24] and [3.25] that

$$
\begin{aligned}
& \gamma=\int_{\lambda_{1}}^{\lambda_{2}}\left(\bar{P}-P_{\mathrm{T}}\right)\left(1+\lambda / r_{0}\right)^{2} d \lambda, \\
& B=\int_{\lambda_{1}}^{\lambda_{2}}\left(P_{\mathrm{T}}-\bar{P}\right)\left(1+\lambda / r_{0}\right) \lambda d \lambda,
\end{aligned}
$$

Eqs. [3.26] are in fact equivalent to Eqs. [1.6] and [1.7], which have been first obtained by Tolman (7).

Boruvka et al. $(24,25)$ follow another (slightly different) way in the thermodynamics of curved interfaces. Instead of the expression

$$
\delta w=\int_{A} \delta w d A=\gamma \delta A+B A \delta H+\Theta A \delta D,
$$

which follows from our Eq. [3.16] for $\zeta=0$ and for an interface of uniform curvature (sphere, cylinder, or plane), they used another equation of the type

$$
\delta w=\gamma^{B N} \delta A+B \delta(A H)+\Theta \delta(A D) .
$$

It is obvious that these authors used another definition of the interfacial (film) tension:

$$
\gamma^{B N}=\gamma-B H-\theta D
$$

One sees that $\gamma^{B N}$ has the meaning of work (per unit area) done during an elementary deformation of the dividing surface at fixed products $A H$ and $A D$. Such a deformation is a combination of dilation, bending, and torsion (cf. Fig. 1). In contrast, the usual interfacial tension, $\gamma$, is connected only with the simple dilation of the surface area (at constant $H$ and $D$ ). That is why, following Refs. (3, 4, 10) we deal with $\gamma$ (instead of $\gamma^{B N}$ ) in the present text.

4. FUNDAMENTAL EQUATIONS OF THE
LOCAL INTERFACIAL THERMODYNAMICS

(a) Micromechanical Derivation

There are two approaches in the interfacial thermodynamics. In one of them Gibbs fun- 
damental equations are postulated for all bulk, surface, line, and even point phases $(4,5,24)$. In the other approach, the interfacial fundamental equations are derived from the fundamental equations of the bulk phases $(4,13$, 26). So far this last approach has been applied rigorously only for flat and spherical interfaces. Our aim below is to extend the second approach to interfaces of arbitrary curvature.

As is known $(2,6)$ the adsorptions of the components in a multicomponent system can be expressed as

$$
\begin{aligned}
\Gamma_{k}=\int_{\lambda_{1}}^{\lambda_{2}}\left(\rho_{k}-\bar{\rho}_{k}\right) \chi d \lambda, & \\
k & =1,2, \ldots, N,
\end{aligned}
$$

where $\rho_{k}$ and

$$
\bar{\rho}_{k}=\rho_{k}^{\mathrm{I}} \theta(-\lambda)+\rho_{k}^{\mathrm{II}} \theta(\lambda)
$$

are the density profiles of the $k$ th component in the real and in the idealized systems. Indeed, in view of Eq. [3.7] one obtains the total interfacial (excess) mass of the $k$ th component by simple integration of Eq. [4.1]:

$$
N_{k}^{s}=\int_{A} \Gamma_{k} d A=\int_{V}\left(\rho_{k}-\bar{\rho}_{k}\right) d V .
$$

If $u$ and $s$ are the local bulk densities of the internal energy and entropy in the real nonhomogeneous system, one can similarly derive expressions for the surface excess densities of these quantities,

$$
\begin{aligned}
& u_{s}=\int_{\lambda_{1}}^{\lambda_{2}}(u-\bar{u}) \chi d \lambda \\
& s_{s}=\int_{\lambda_{1}}^{\lambda_{2}}(s-\bar{s}) \chi d \lambda
\end{aligned}
$$

with

$$
\begin{gathered}
\bar{u}=u^{\mathrm{I}} \theta(-\lambda)+u^{\mathrm{II}} \theta(\lambda), \\
\bar{s}=s^{\mathrm{I}} \theta(-\lambda)+s^{\mathrm{II}} \theta(\lambda) .
\end{gathered}
$$

As usual, the superscripts I and II denote the values of the respective quantity in the two phases of the idealized system. As far as we know, the expressions [4.1] and [4.3] have been first obtained by Murphy (2). Our purpose below is to derive thermodynamic relationships between $u_{s}, s_{s}, \Gamma_{k}(k=1,2, \ldots, N)$ from the Gibbs fundamental equations in the bulk phases. For the sake of simplicity we will restrict ourselves only to slow equilibrium processes, for which the temperature, $T$, and the chemical potentials, $\mu_{k}$, of the soluble components are constant throughout the system. It is not necessary to presume that the chemical potentials of the insoluble components (present only at the interface) are constant throughout the dividing surface.

Let $\tilde{u}, \tilde{s}$, and $x_{k}$ be the local (bulk) mass densities of internal energy, entropy, and mass of the $k$ th component:

$$
\begin{gathered}
\tilde{u}=u / \rho, \quad \tilde{s}=s / \rho, \quad x_{k}=\rho_{k} / \rho, \\
k=1,2, \ldots, N .
\end{gathered}
$$

The Gibbs fundamental equation of a nonisotropic material continuum is usually written in the form (20)

$$
\delta \tilde{u}=T \delta \tilde{s}-\frac{1}{\rho} \underline{\mathbf{P}}: \underline{\Phi} \delta t+\sum_{k=1}^{N} \mu_{k} \delta x_{k} .
$$

Here $\delta \tilde{u}$ stands for $(d \tilde{u} / d t) \delta t$ (the same for $\delta \tilde{s}$ and $\delta x_{k}$ ). Then by means of the continuity equation

$$
\frac{d \rho}{d t}+\rho \nabla \cdot \mathbf{w}=0
$$

and of Eq. [4.5] one can easily transform Eq. [4.6] as follows:

$$
\begin{aligned}
\frac{d u}{d t}+u \nabla \cdot \mathbf{w} & =\mathrm{T}\left(\frac{d s}{d t}+s \nabla \cdot \mathbf{w}\right) \\
& -\underline{\mathbf{P}}: \underline{\mathbf{\Phi}}+\sum_{k=1}^{N} \mu_{k}\left(\frac{d \rho_{k}}{d t}+\rho_{k} \nabla \cdot \mathbf{w}\right) .
\end{aligned}
$$

On the other hand, it was proven by Eliassen (Ref. (17), Eq. [D.2.18]) that

$$
\frac{d \chi}{d t}=\chi\left(\nabla \cdot \mathbf{w}-\nabla_{\mathrm{II}} \cdot \mathbf{v}\right)
$$


By means of Eq. [4.8] one can easily prove that the equation

$$
\chi\left(\frac{d u}{d t}+u \nabla \cdot \mathbf{w}\right)=\frac{d(u \chi)}{d t}+u \chi \nabla_{\mathrm{II}} \cdot \mathbf{v}
$$

is identically satisfied. In the idealized system instead of Eqs. [4.7] and [4.9] one has

$$
\begin{aligned}
& \frac{d u^{Y}}{d t}+u^{Y} \nabla \cdot \mathbf{w}=\mathrm{T}\left(\frac{d s^{Y}}{d t}+s^{Y} \nabla \cdot \mathbf{w}\right) \\
& -\underline{\mathbf{P}}^{Y}: \underline{\mathbf{\Phi}}+\sum_{k=1}^{N} \mu_{k}\left(\frac{d \rho_{k}^{Y}}{d t}+\rho_{k}^{Y} \nabla \cdot \mathbf{w}\right),
\end{aligned}
$$$$
Y=\mathrm{I}, \mathrm{II}, \quad[4.10]
$$

and

$$
\begin{aligned}
\chi\left(\frac{d u^{Y}}{d t}+u^{Y} \nabla \cdot \mathbf{w}\right) \\
=\frac{d\left(u^{Y} \chi\right)}{d t}+u^{Y} \chi \nabla_{\mathrm{II}} \cdot \mathbf{v}
\end{aligned}
$$

Then from Eqs. [4.4] and [4.11] one obtains

$$
\begin{aligned}
\chi\left[\theta(-\lambda) \frac{d u^{\mathrm{I}}}{d t}\right. & \left.+\theta(\lambda) \frac{d u^{\mathrm{II}}}{d t}+\bar{u} \nabla \cdot \mathbf{w}\right] \\
& =\frac{d(\bar{u} \chi)}{d t}+\bar{u} \chi \nabla_{\mathrm{II}} \cdot \mathbf{v} .
\end{aligned}
$$

The subtraction of Eq. [4.12] from Eq. [4.9], followed by integration with respect to $\lambda$, leads to

$$
\begin{gathered}
\int_{\lambda_{1}}^{\lambda_{2}} d \lambda \chi\left[\frac{d u}{d t}-\theta(-\lambda) \frac{d u^{\mathrm{I}}}{d t}-\theta(\lambda) \frac{d u^{\mathrm{II}}}{d t}\right. \\
+(u-\bar{u}) \nabla \cdot \mathbf{w}]=\frac{d u_{s}}{d t}+u_{s} \nabla_{\mathrm{II}} \cdot \mathbf{v}
\end{gathered}
$$

(see also Eq. [4.3]).

Equations of the type of Eq. [4.13] can be derived also for the entropy and mass densities, $s$ and $\rho_{k}, k=1,2, \ldots, N$. Then from Eqs. [4.7] and [4.9] one easily derives

$$
\begin{aligned}
\frac{d u_{s}}{d t}+u_{s} \dot{\alpha}= & T\left(\frac{d s_{s}}{d t}+s_{s} \dot{\alpha}\right)-\int_{\lambda_{1}}^{\lambda_{2}} \underline{\mathbf{P}}^{\chi}: \underline{\Phi} d \lambda \\
& +\sum_{k=1}^{N} \mu_{k}\left(\frac{d \Gamma_{k}}{d t}+\Gamma_{k} \dot{\alpha}\right), \quad[4.14]
\end{aligned}
$$

where Eqs. [2.16], [3.9], [4.1], and [4.3] have been used. The multiplication of Eq. [4.14] by $\delta t$ in view of Eqs. [3.11], [3.16], and [3.17] leads to the interfacial fundamental equation

$$
\begin{aligned}
\delta u_{s}=T \delta s_{s}+ & \sum_{k=1}^{N} \mu_{k} \delta \Gamma_{k}+\left(\gamma-\omega_{s}\right) \delta \alpha \\
& +\zeta \delta \beta+B \delta H+\Theta \delta D
\end{aligned}
$$

where

$$
\omega_{s}=u_{s}-T s_{s}-\sum_{k=1}^{N} \mu_{k} \Gamma_{k}
$$

is the interfacial density of the grand thermodynamical potential. If the interface is an ideal two-dimensional fluid and the adsorbed matter is soluble in the bulk phases, then $\gamma$ $=\omega_{s}, \zeta=0$, and Eq. [4.16] transforms into (6)

$$
\delta u_{s}=T \delta s_{s}+\sum_{k=1}^{N} \mu_{k} \delta \Gamma_{k}+B \delta H+\Theta \delta D
$$

The interfacial density of the free energy is

$$
f_{s}=u_{s}-T S_{s}=\omega_{s}+\sum_{k=1}^{N} \mu_{k} \Gamma_{k} .
$$

Then the fundamental equation [4.15] can be rewritten in the form

$$
\begin{aligned}
\delta f_{s}=-s_{s} \delta T+ & \sum_{k=1}^{N} \mu_{k} \delta \Gamma_{k}+\left(\gamma-\omega_{s}\right) \delta \alpha \\
& +\zeta \delta \beta+B \delta H+\Theta \delta D
\end{aligned}
$$

\section{(b) Discussion}

If all components present at the interface are soluble at least in one of the neighboring bulk phases, then $\gamma=\omega_{s}$ and the term with $\delta \alpha$ in Eqs. [4.15] and [4.19] will disappear. However, if there are insoluble components at the interface, which are not in contact with a bulk reservoir containing these components, then the respective variations $\delta \Gamma_{k}$ should be connected somehow with the interfacial dilation $\delta \alpha$. Some additional information can be 
gained by means of the interfacial mass balance equation of an insoluble component

$$
\frac{d \Gamma_{k}}{d t}+\Gamma_{k} \nabla_{\mathrm{II}} \cdot \mathbf{v}=-\nabla_{\mathrm{II}} \cdot \mathbf{I}_{k}^{s}
$$

where $\mathbf{I}_{k}^{s}=\Gamma_{k}\left(\mathbf{v}_{k}-\mathbf{v}\right)$ is the mass current due to surface difusion with $\mathbf{v}_{k}$ being the velocity of the $k$ th surface component. We deal with an insoluble adsorbed component and that is why we have omitted the contribution of the mass exchange between the bulk and the interface in Eq. [4.20]. It is known that the righthand side of Eq. [4.20] represents the mass current due to the surface diffusion. Let us multiply this equation by $\delta t$. In view of Eqs. [2.16] and [3.17] we obtain

$$
\delta \Gamma_{k}=-\Gamma_{k} \delta \alpha-\delta \Lambda_{k},
$$

where

$$
\delta \Lambda_{k}=\left(\nabla_{\mathrm{II}} \cdot \mathbf{I}_{k}^{s}\right) \delta t=\left\{\nabla_{\mathrm{II}} \cdot\left[\Gamma_{k}\left(\mathbf{v}_{k}-\mathbf{v}\right)\right]\right\} \delta t .
$$

$\delta \Lambda_{k}$ is the elementary amount of matter transported by surface diffusion. The experiments show $(11,27,28)$ that a change in the shape of the interface can produce redistribution of the adsorbed matter: depending on their shape the molecules of some adsorbed components prefer to occupy those parts of the area which have positive values of the mean curvature, $H$, whereas the molecules of other components concentrate around places with negative $H$. Therefore, a deformation, which causes extension of one of the adsorbed components could lead to compression of another adsorbed component. This redistribution gives rise to the increment $\delta \Lambda_{k}$ even for quasistatic processes.

When the composition of the insoluble surface mixture is uniform throughout the interface (i.e., the composition does not depend on the local curvature), then $\delta \Lambda_{k}=0$ and one obtains from Eq. [4.21]

$$
\begin{gathered}
\delta \Gamma_{k}=-\Gamma_{k} \delta \alpha, \\
k=M+1, M+2, \ldots, N .
\end{gathered}
$$

It is supposed here that the adsorbed components with $k=M+1, M+2, \ldots, N$ are insoluble in the bulk phases. In view of Eq. [4.22] one can transform Eq. [4.19] to read

$$
\begin{aligned}
\delta \hat{\omega}_{s}=-S_{s} \delta T & -\sum_{k=1}^{M} \Gamma_{k} \delta \mu_{k}+\left(\gamma-\hat{\omega}_{s}\right) \delta \alpha \\
& +\zeta \delta \beta+B \delta H+\Theta \delta D, \quad
\end{aligned}
$$

where

$\hat{\omega}_{s}=\omega_{s}+\sum_{k=M+1}^{N} \Gamma_{k} \mu_{k}=f_{s}-\sum_{k=1}^{M} \Gamma_{k} \mu_{k}$

is the surface density of the modified grand potential (cf. Eq. [4.18]). The chemical potentials of the soluble components are appropriate parameters of the thermodynamic state, because they are determined by the composition in the bulk phases. In particular, one can derive from Eq. [4.23] a version of the Shuttleworth $(20,29)$ equation:

$$
\gamma=\hat{\omega}_{s}+\left(\frac{\partial \hat{\omega}_{s}}{\partial \alpha}\right)_{T, \beta, H, D, \mu_{1}, \ldots, \mu_{M}} .
$$

The derivative in Eq. [4.25] is zero only at equilibrium spreading (contact with reservoir of the insoluble adsorbed component) or when there are no adsorbed insoluble components at all (see Refs. $(30,31)$ ).

\section{CONDITIONS FOR INTERFACIAL MECHANICAL EQUILIBRIUM}

\section{(a) Derivation by Variational Principle}

The integral excess interfacial free energy and its first variation are

$$
\begin{gathered}
F^{s}=\int_{A} f_{s} d A, \\
\delta F^{s}=\delta \int_{A} f_{s} d A=\int_{A}\left(\delta f_{s}+f_{s} \delta \alpha\right) d A,
\end{gathered}
$$

where we used the fact that $\delta \alpha x=\delta(d A) / d A$. The substitution of $f_{s}$ and $\delta f_{s}$ from Eqs. [4.18] and [4.19], and the assumption that the temperature and the chemical potentials as well as their variations are uniform throughout the interface, yield 


$$
\begin{aligned}
& \delta F^{s}=-S^{s} \delta T+\sum_{k=1}^{N} \mu_{k} \delta N_{k} \\
& +\int_{A}(\gamma \delta \alpha+\zeta \delta \beta+B \delta H+\Theta \delta D) d A,[5.1]
\end{aligned}
$$

where

$$
\begin{array}{r}
S^{s}=\int_{A} s^{s} d A \text { and } \quad N_{k}^{s}=\int_{A} \Gamma_{k} d A, \\
k=1,2, \ldots, N
\end{array}
$$

are the total interfacial amounts of entropy and mass of the different components. The variation in the free energy of the two bulk phases of the idealized system is

$$
\begin{aligned}
\delta F^{b}=-\left(S^{\mathrm{I}}+S^{\mathrm{II}}\right) & \delta T+\sum_{k=1}^{N} \mu_{k} \delta\left(N_{k}^{\mathrm{I}}+N_{k}^{\mathrm{II}}\right) \\
& -P^{\mathrm{I}} \delta V^{\mathrm{I}}-P^{\mathrm{II}} \delta V^{\mathrm{II}},
\end{aligned}
$$

where $S^{Y}, N_{k}^{Y}, V^{Y}(Y=1$, II $)$ stand for the entropy, the mass of the $k$ th component, and the volume of the bulk phase $Y$. The total amounts of the respective quantities for all the system are

$$
\begin{gathered}
F=F^{b}+F^{s}, \quad S=S^{\mathrm{I}}+S^{\mathrm{II}}+S^{s}, \\
V=V^{\mathrm{I}}+V^{\mathrm{II}}, \\
N_{k}=N_{k}^{\mathrm{I}}+N_{k}^{\mathrm{II}}+N_{k}^{s}, \quad k=1,2, \ldots, N .
\end{gathered}
$$

Then the summation of Eqs. [5.1] and [5.3] yields

$$
\begin{aligned}
\delta F & =-S \delta T+\sum_{k=1}^{N} \mu_{k} \delta N_{k} \\
& -\left(P^{\mathrm{I}}-P^{\mathrm{II}}\right) \delta V^{\mathrm{I}}-P^{\mathrm{II}} \delta V+\int_{A} \\
& \times(\gamma \delta \alpha+\zeta \delta \beta+B \delta H+\Theta \delta D) d A .
\end{aligned}
$$

When deriving Eq. [5.4] we supposed that all chemical potentials are uniform throughout the system; if some components are present only in the film (interface), their chemical potentials are uniform throughout the film (interface).

Now, let the elementary process in the system be a deformation of the interface

$$
\delta \mathbf{R}=\zeta^{\mu} \mathbf{a}_{\mu}+\eta \mathbf{n}
$$

( $R$ is the running position vector of the dividing surface and $n$ is its unit running normal) at fixed boundaries

$$
\zeta^{1}=\zeta^{2}=\eta=0,
$$

$$
\eta_{, \mu}=0 \quad \text { over the contour } C
$$

$\left(\zeta^{\mu}=v^{\mu} \delta t\right.$ and $\eta=v^{[n]} \delta t$ are infinitesimal quantities and $C$ is the contour comprising the surface ). $T, V$, and $N_{k}(k=1,2, \ldots, N)$ are constant during such a process and hence

$$
\begin{aligned}
& \delta F=-\left(P^{\mathrm{I}}-P^{\mathrm{II}}\right) \int_{A} \eta d A \\
& +\int_{A}(\gamma \delta \alpha+\zeta \delta \beta+B \delta H+\Theta \delta D) d A
\end{aligned}
$$

By multiplication of Eqs. [2.11], [2.12], [2.26], and [2.27] by $\delta t$ one obtains

$$
\begin{gathered}
\delta \alpha=a^{\mu \sigma} \zeta_{\mu, \sigma}-2 H \eta, \\
\delta \beta=q^{\mu \sigma} \zeta_{\mu, \sigma}-2 D \eta, \\
\delta H=H_{, \mu} \zeta^{\mu}+\left(H^{2}+D^{2}\right) \eta+\frac{1}{2} a^{\mu \sigma} \eta_{, \mu \sigma}, \\
\delta D=D_{, \mu} \zeta^{\mu}+2 H D \eta+\frac{1}{2} q^{\mu \sigma} \eta_{, \mu \sigma} .
\end{gathered}
$$

These equations in conjunction with the equilibrium condition $\delta F=0$ yield

$$
\begin{aligned}
0= & \int_{A}\left[\left(\gamma a^{\mu \sigma}+\zeta q^{\mu \sigma}\right) \zeta_{\mu, \sigma}\right. \\
& \left.+\left(B H_{, \mu}+\Theta D_{, \mu}\right) \zeta^{\mu}\right] d A \\
& +\int_{A}\left\{\left[P^{\mathrm{II}}-P^{\mathrm{I}}-2 H \gamma-2 D \zeta\right.\right. \\
& \left.+\left(H^{2}+D^{2}\right) B+2 H D \theta\right] \eta \\
& \left.\quad+\frac{1}{2}\left(B a^{\mu \sigma}+\Theta q^{\mu \sigma}\right) \eta_{, \mu \sigma}\right\} d A .
\end{aligned}
$$

Using the known Green theorem (19) one can represent the surface integrals in Eq. [5.8], which contain the derivatives $\zeta_{\mu, \sigma}$ and $\eta_{, \mu \sigma}$ in the integrand, as sums of surface integrals containing $\zeta_{\mu}$ and $\eta$, and line integrals over the contour $C$. The integrals over $C$ are equal to zero due to Eq. [5.6]. Moreover, the variations $\zeta_{\mu}(\mu=1,2)$ and $\eta$ are independent. Then by setting the coefficients before $\zeta_{\mu}(\mu=1,2)$ and 
$\eta$ equal to zero one finally obtains the soughtfor conditions for mechanical equilibrium:

$$
\begin{aligned}
& \gamma_{, \mu}+\zeta_{, \sigma} q_{\mu}^{\sigma}=B H_{, \mu}+\Theta D_{, \mu}, \quad \mu=1,2 ;[5.9] \\
& -2 H \gamma-2 D \zeta+\left(H^{2}+D^{2}\right) B+2 H D \Theta \\
& \quad+\frac{1}{2}\left(a^{\mu \sigma} B_{, \mu \sigma}+q^{\mu \sigma} \Theta_{, \mu \sigma}\right)=P^{\mathrm{I}}-P^{\mathrm{II}} \quad[5.10]
\end{aligned}
$$

which must hold in each point of the dividing surface. More precisely, Eq. [5.11] is a condition for tangential and Eq. [5.12] for normal equilibrium. (Murphy (2) has erroneously considered $\eta$ and $\eta_{, \mu \sigma}$ as being independent variations and thus the number of equations obtained by him is greater than the real number of the independent variations.)

\section{(b) Discussion}

Equation [5.10] is a generalized form of the Laplace equation [1.4]. Except for the last term in the left-hand side of Eq. [5.10] it was obtained by Buff $(9,10)$ from a phenomenological expression for the surface stress tensor postulated by him. Indeed, by means of Eqs. [1.2], [3.21], and [3.22] Eq. [5.10] (irrespective of the term with the second derivatives) can be transformed to read

$$
-c_{1} \gamma_{1}-c_{2} \gamma_{2}+c_{1}^{2} B_{1}+c_{2}^{2} B_{2}=P^{\mathrm{I}}-P^{\mathrm{II}},
$$

which is the result of $\operatorname{Buff}(9,10)$. (Note that in Refs. $(9,10)$ Buff accounts also for the weight of the interface, which is neglected here.) However, the tangential balance in Ref. (10)

$$
(\gamma-B H)_{, \mu}=0, \quad \mu=1,2,
$$

differs significantly from our expression (Eq. [5.9]). This could mean that the phenomenological surface stress tensor postulated in Ref. (10) is not entirely correct.

At the surface of tension (see Ref. (6)) $B$ $=\theta=0$ and the balance equations [5.9] and [5.10] considerably simplify:

$$
\begin{gathered}
\left(\gamma a^{\mu \sigma}+\zeta q^{\mu \sigma}\right)_{, \sigma}=0, \quad \mu=1,2 \\
-2 H \gamma-2 D \zeta=P^{\mathrm{I}}-P^{\mathrm{II}}
\end{gathered}
$$

If the coordinate lines are chosen to be the lines of curvature, from Eqs. [2.7], [3.21], and [5.11] one obtains

$$
\left(\gamma_{1}\right)_{, 1}=0, \quad\left(\gamma_{2}\right)_{, 2}=0
$$

which means that one of the $\gamma_{i}$ 's $(i=1,2)$ must be constant along the lines of maximum curvature and the other must be constant along the lines of minimum curvature. Moreover, $c_{i}=-1 / R_{i}(i=1,2)$, where $R_{1}$ and $R_{2}$ are the two principal radii of curvature. Then Eq. [5.12] along with Eq. [1.2] leads to

$$
\begin{array}{r}
\gamma\left(1 / R_{1}+1 / R_{2}\right)+\zeta\left(1 / R_{1}-1 / R_{2}\right) \\
=P^{\mathrm{I}}-P^{\mathrm{II}} .
\end{array}
$$

If the shearing tension $\zeta$ is zero (the interface is a two-dimensional fluid), Eq. [5.14] reduces to the usual Laplace equation. In addition, $\zeta$ $=0$ yields $\gamma_{1}=\gamma_{2}=\gamma$ and then Eq. [5.13] shows that in this case $\gamma$ must be constant throughout the whole interface.

\section{CONCLUDING REMARKS}

An investigation of the interfacial rate-ofstrain tensor, $\underline{\mathbf{d}}$, and of the mechanical work for interfacial deformation is presented in this paper. The interfacial shear deformation was characterized by a scalar parameter $\beta$. The important special case, when the tensor $\underline{\mathbf{d}}$ is diagonal in the basis of the principal curvatures, was considered in more detail. The interfacial stretching and shearing tensions, $\gamma$ and $\zeta$, and the interfacial bending and torsion moments, $B$ and $\Theta$, were expressed as integrals over the components of the pressure tensor. These expressions are more general than the respective equations of Buff (10) and Murphy (2).

The Gibbs fundamental equations of an interface are derived in Section 4 from the fundamental equations of the bulk phases. Then by variation of the total free energy of the system the interfacial conditions for mechanical equilibrium which generalize the equations obtained earlier by Buff ( 10$)$ are derived. The normal balance equation reduces to the known 
Laplace equation when $B=\theta=0$. The last relation is satisfied at a special dividing surface, the so-called "surface of tension."

\section{APPENDIX}

In view of Eqs. [2.10] and [3.4] one has

$$
\begin{gathered}
\mathbf{w}=\mathbf{a}^{\mu} \mathrm{W}_{\mu}+\mathbf{n} v^{[n]}, \\
w_{\mu}=v_{\mu}-\lambda\left(b_{\mu}^{\sigma} v_{\sigma}+v_{, \mu}^{[n]}\right) .
\end{gathered}
$$

The surface gradient of the space vector $w$ is (17)

$$
\begin{aligned}
\nabla_{\text {II }} \mathbf{w}=\mathbf{a}^{\mu} \mathbf{a}^{\nu}\left(w_{\nu, \mu}-b_{\mu \nu} v^{[n]}\right) & \\
& +\mathbf{a}^{\mu} \mathbf{n}\left(b_{\mu}^{\sigma} w_{\sigma}+v_{, \mu}^{[n]}\right) .
\end{aligned}
$$

Moreover, the space gradient operator can be represented in the form (17)

$$
\nabla=\frac{1}{\chi} \underline{\mathbf{L}} \cdot \nabla_{\mathrm{II}}+\mathbf{n} \frac{\partial}{\partial \lambda}
$$

(cf. Eqs. [3.6] and [3.7]). Then

$$
\begin{aligned}
\nabla \mathbf{w}= & \frac{1}{\chi}\left[(1-2 \lambda H) a_{\mu \nu}+\lambda b_{\mu \nu}\right] \mathbf{a}^{\mu} \mathbf{a}^{\nu} \\
& \times \nabla_{\Pi} \mathbf{w}+\mathbf{n} \frac{\partial \mathbf{w}}{\partial \lambda} \\
= & \mathbf{a}^{\mu} \mathbf{a}^{\nu}\left[(1-2 \lambda H) w_{\nu, \mu}\right. \\
& -(1-2 \lambda H) b_{\mu \nu} v^{[n]}+\lambda b_{\mu}^{\sigma} w_{\nu, \sigma} \\
& \left.-\lambda b_{\mu}^{\sigma} b_{\sigma, \nu} v^{[n]}\right] / \chi+\mathbf{a}^{\mu} \mathbf{n}[(1-2 \lambda H) \\
& \times b_{\mu}^{\sigma} w_{\sigma}+(1-2 \lambda H) v_{, \mu}^{[n]} \\
& \left.+\lambda b_{\mu}^{v} b_{\nu}^{\sigma} w_{\sigma}+\lambda b_{\mu}^{\sigma} v_{, \sigma}^{[n]}\right] / \chi \\
& \quad-\mathbf{n} \mathbf{a}^{\mu}\left[b_{\mu}^{\sigma} v_{\sigma}+v_{, \mu}^{[n]}\right] . \quad[\mathrm{A} .2]
\end{aligned}
$$

The substitution of Eq. [A.2] in Eq. [3.3] along with Eq. [3.7] and the Gauss equation $b_{\mu}^{\sigma} b_{\sigma}^{v}=2 H b_{\mu}^{\nu}-K \delta_{\mu}^{\nu}$ leads to Eq. [3.8].

By means of Eqs. [2.7], [2.11], [2.12], [2.26], [2.27], [A.1], and the identity $b_{\mu v, \sigma}$ $=b_{\mu \sigma, v}$, one obtains that

$$
\begin{aligned}
A_{1}=a^{\mu \nu} w_{\mu, \nu}=\dot{\alpha}+2 H v^{[n]} & \\
& -\lambda(H \dot{\alpha}+D \dot{\beta}+2 \dot{H})
\end{aligned}
$$

$$
\begin{gathered}
A_{2}=q^{\mu v} w_{\mu, \nu}=\dot{\beta}+2 D v^{[n]} \\
\quad-\lambda(H \dot{\beta}+D \dot{\alpha}+2 \dot{D}) \\
\bar{B}_{1}=\frac{1}{2} a^{\mu \nu}\left(w_{\mu, \sigma} b_{\nu}^{\sigma}+w_{\nu, \sigma} b_{\mu}^{\sigma}\right)=H A_{1}+D A_{2} \\
\bar{B}_{2}=\frac{1}{2} q^{\mu \nu}\left(w_{\mu, \sigma} b_{\nu}^{\sigma}+w_{\nu, \sigma} b_{\mu}^{\sigma}\right)=D A_{1}+H A_{2} \\
C_{1}=a^{\mu \nu}\left(b_{\mu \nu}-\lambda K a_{\mu \nu}\right) v^{[n]}=2(H-\lambda K) v^{[n]} \\
C_{2}=q^{\mu \nu}\left(b_{\mu \nu}-\lambda K a_{\mu \nu}\right) v^{[n]}=2 D v^{[n]} .
\end{gathered}
$$

Then it follows from Eq. [3.8] that

$$
\begin{aligned}
a^{\mu \nu} \Phi_{\mu \nu} & =\frac{1}{\chi}\left[(1-2 \lambda H) A_{1}+\lambda \bar{B}_{1}-C_{1}\right] \\
q^{\mu \nu} \Phi_{\mu \nu} & =\frac{1}{\chi}\left[(1-2 \lambda H) A_{2}+\lambda \bar{B}_{2}-C_{2}\right],
\end{aligned}
$$

where $\Phi_{\mu \nu}=\mathbf{a}_{\mu} \cdot \underline{\Phi} \cdot \mathbf{a}_{\nu}$. Moreover, $\underline{\mathbf{U}}_{\mathrm{II}}=a^{\mu \nu}$ $\times \mathbf{a}_{\mu} \mathbf{a}_{\nu}, \mathbf{q}=q^{\mu \nu} \mathbf{a}_{\mu} \mathbf{a}_{\nu}, \underline{\mathbf{L}}=(1-2 \lambda H) \underline{\mathbf{U}}_{\mathrm{II}}+\lambda D \mathbf{q}$, and one can derive Eq. [3.15] from Eq. [A.3] by means of some simple but time-consuming calculations.

\section{ACKNOWLEDGMENTS}

The work is supported by the Committee for Science of Bulgaria. The author is indebted to Referee A for his valuable comments.

\section{REFERENCES}

1. Gibbs, J. W., "The Scientific Papers of J. Willard Gibbs," Vol. I. Dover, New York, 1961.

2. Murphy, C. L., PhD thesis, University of Minnesota, 1966. University Microfilms, Ann Arbor, Michigan, 1984.

3. Kondo, S., J. Chem. Phys. 25, 662 (1956).

4. Ono, S., and Kondo, S., "Molecular Theory of Surface Tension in Liquids," in "Handbuch der Physik," Vol. X. Springer, Berlin, 1960.

5. Rusanov, A. I., "Phase Equilibria and Surface Phenomena." Khimia, Leningrad, 1967 [in Russian]; "Phasengleichgewichte und Grenzflachenerscheinungen.' Akademie Verlag, Berlin, 1978.

6. Ivanov, I. B., and Kralchevsky, P. A., in "Thin Liquid Films" (I. B. Ivanov, Ed.), p. 49. Dekker, New York, 1988.

7. Tolman, R. C., J. Chem. Phys. 16, 758 (1948).

8. Buff, F. P., J. Chem. Phys. 23, 419 (1955).

9. Buff, F. P., "The Theory of Capillarity," in "Handbuch der Physik," Vol. X, p. 281. Springer, Berlin, 1960.

10. Buff, F. P., Discuss. Faraday Soc. 30, 52 (1960).

11. Evans, A. E., and Skalak, R., CRC Critical Rev. Bioeng. 3, 181 (1979). 
12. Good, R. J., and Buff, F. P., in "The Modern Theory of Capillarity" (F. C. Goodrich and A. I. Rusanov, Eds.). Akademie Verlag, Berlin, 1981.

13. Ivanov, I. B., and Toshev, B. V., Colloid Polym. Sci. 253, 593 (1975).

14. Kralchevsky, P. A., and Ivanov, I. B., in "Surfactants in Solution" (K. L. Mittal, Ed.), p. 1549. Plenum, New York, 1986.

15. Scriven, L. E., Chem. Eng. Sci. 12, 98 (1960).

16. Aris, R., "Vectors, Tensors and Basic Equations of Fluid Mechanics." Prentice-Hall, New York, 1962.

17. Eliassen, J. D., PhD thesis, University of Minnesota, 1963. University Microfilms, Ann Arbor, MI, 1983.

18. Weatherburn, C. E., "Differential Geometry of Three Dimensions," Vols. I and II. University Press, Cambridge, 1939.

19. McConnell, A. J., "Application of Tensor Analysis." Dover, New York, 1957.

20. Podstrigach, Y. S., and Povstenko, Y. Z., "Introduction in Mechanics of Surface Phenomena in De- formable Solids." Naukova Dumka, Kiev, 1985 [in Russian].

21. Gurkov, T. D., and Kralchevsky, P. A., J. Surface Sci. Technol., submitted for publication.

22. Buff, F. P., J. Chem. Phys. 25, 146 (1956).

23. Rowlinson, J. S., and Widom, B., "Molecular Theory of Capillarity." Clarendon, Oxford, 1982.

24. Boruvka, L., and Newmann, A. W., J. Chem. Phys. 66, 5464 (1977).

25. Boruvka, L., Rotenberg, Y., and Newmann, A. W., J. Phys. Chem. 89, 2714 (1985).

26. Toshev, B. V., and Ivanov, I. B., Colloid Polym. Sci. 253, 558 (1975).

27. Markin, V., and Glaser, R., Stud. Biophys. 80, 201 (1980).

28. Markin, V. S., Biophys. J. 36, 1 (1981).

29. Shuttleworth, R., J. Phys. A 63, 444 (1950).

30. Eriksson, J. C., J. Colloid Interface Sci. 37, 659 (1971).

31. Benson, G. C., and Yun, K. S., in "The Solid-Gas Interface" (E. A. Flood, Ed.), Vol. 1, p. 203. Dekker, New York, 1967. 